\title{
Chapter 1 \\ Biosecurity for Synthetic Biology and Emerging Biotechnologies: Critical Challenges for Governance
}

\author{
Benjamin D. Trump, Marie-Valentine Florin, Edward Perkins, \\ and Igor Linkov
}

\subsection{An Introduction to the Dual-Use Nature of Emerging Biotechnology}

Synthetic biology uses engineering-based modeling and building techniques to modify existing organisms and microbes or to construct them from scratch. The rate of development and research related to synthetic biology for both industry and academia has increased over the past two decades (Ahteensuu 2017), with applications in medicine (new vaccines, delivery of therapeutics, and treatments), energy (biofuels), environmental remediation, food production, and general industry (detergents, adhesives, perfumes) (Evans and Selgelid 2015; Gronvall 2015).

While synthetic biology heralds advances in these fields, its techniques could also be adapted for malicious purposes and used by terrorist organizations, rogue actors, or hostile nations to create dangerous pathogens, invasive organisms, or other disruptive biological agents (Yeh et al. 2012). Such potential makes synthetic biology a dual-use research area of concern (DURC) since the same techniques can be used to benefit or harm people, animals, environments, or nations (Getz and

\footnotetext{
B. D. Trump $(\bowtie) \cdot$ I. Linkov

US Army Corps of Engineers, Engineer Research and Development Center,

Concord, MA, USA

e-mail: Benjamin.D.Trump@usace.army.mil; igor.linkov@usace.army.mil

M.-V. Florin

International Risk Governance Center, École Polytechnique Fédérale de Lausanne,

Lausanne, Switzerland

e-mail: Marie-Valentine.Florin@epfl.ch

E. Perkins

US Army Corp of Engineers, Engineer Research and Development Center,

Vicksburg, MS, USA

e-mail: edward.j.perkins@usace.army.mil
} 
Dellaire 2018). Indeed, there have been 35 confirmed cases of biological weapons deployment between 1970 and 2014 (Franconi et al. 2018). Thus far, the use of advanced biotechnologies for weapons production has mostly been pursued by state-actors. However, synthetic biological weapons are expected to become a larger concern as the field advances and they become increasingly adopted by malicious sub-state or non-state actors (Gronvall 2018).

Synthetic biology raises the possibility that pathogenic bioweapons could be designed, developed, and deployed in new ways that diverge from the diseasecausing characteristics of naturally occurring pathogens (NAS 2018). Traditionally, only known pathogens found naturally in the environment, such as B. anthracis and $Y$. pestis, were developed as biological weapons because of the inherent infectious characteristics that readily enabled such organisms to serve as weapons. However, as synthetic biology continues to expand its capabilities to create and modify biological weapons, there is an increasing need for biosafety and biosecurity assurances for humans, animals, plants, and the environment. To cope with threats arising from synthetic biology's dual-use nature, biosecurity is needed to prevent, detect, and determine the source of biological attacks.

Biological weapons created from synthetic biology represent a new and unique threat space. Potential threats from synthetic biology include increased pathogen transmissibility between and within species in addition to resistance to established treatments. Synthetic biology can be used to engineer normally benign microbes that produce toxic biological compounds or re-build extinct or hard-to-obtain pathogens from scratch (NAS 2018). The main biotechnologies of concern in the near future (over the course of the next decade or so) are projected to be oligonucleotide synthesis, DNA assembly (assembling multiple smaller fragments of oligonucleotides into the desired larger sequence), and genetic modification (editing, deleting, and inserting desired sequences into targeted sites of a genome). Harm may also arise through the purposeful use of these techniques to disrupt human and environmental systems. Malevolent use, but also negligent use (misuse) of synthetic biology techniques, require two circumstances: (a) the spread of information, techniques, or knowhow to utilize synthetic biology's enabling technologies for irresponsible or nefarious purposes ("information hazard"), and (b) the ability to use such knowledge and tools to generate and disseminate harmful engineered organisms to vulnerable recipients.

Creating effective biosecurity procedures and policies to protect humans, agriculture, technology, and the environment from such nefarious usages (or accidental or negligent damage from misuse) will require understanding the current state of synthetic biology. This includes knowing the platforms and technologies available for manipulation or construction (e.g., viruses, microbes, multi-cellular organisms, or cell-free systems) and planning for the future as the field overcomes identified bottlenecks or roadblocks. Biosecurity will require developing screening mechanisms for synthetic pathogens and biological attacks, as well as methods to assess how a synthetic pathogen could be dispersed during an attack, identify what the potential targets of the attack are, and identify who developed the bioweapon. 


\subsection{History}

State-sponsored biological warfare has been employed since ancient times (Mayor 2003). By 1925, germ theory and advances in bacteriology had led to enough advancement in the field that regulation was deemed necessary, leading to the Geneva Protocol, which banned the use of chemical and biological weapons in war. However, this did not prevent a number of countries from embarking on biological weapons programs. A notorious usage occurred during World War II, when Japanese units deployed bombs filled with plague-ridden fleas in China as a part of their biological weapons program. Other nations also invested in biological weapons programs, including the UK and the US, although they did not end up deploying the weapons they developed. These state-based biological weapons capabilities tended to be justified as "first strike" options or as methods to weaken enemy ground forces that could not be combatted through conventional ordnance. In all cases, states developed the scientific and material ability to build, package, and deploy biological weapons.

A major regulatory development came in 1972, with the passing of the Convention on the Prohibition of the Development, Production and Stockpiling of Bacteriological (Biological) and Toxin Weapons and on Their Destruction (usually referred to as the Biological Weapons Convention; here "BWC"), an update to the Geneva Protocol. The BWC banned the "development, production and stockpiling of microbes or their poisonous products except in amounts necessary for protective and peaceful research" ("Convention," 1972). The BWC went further than the Geneva Protocol in banning not just the use of biological weapons in war, but also their development and possession. Although 183 countries are parties to the BWC, some countries with a known interest in biological weapons, such as Israel, have not signed or ratified the BWC, and some countries that have ratified the BWC, such as Russia and China, have completely or partially ignored its statutes. Russia had an active biological weapons program comprising tens of facilities and thousands of researchers until at least 1992, twenty years after it signed the BWC (Zilinskas 2012). China is believed to have aided Iran's biological weapons program through the sale of sensitive dual-use equipment and vaccines since at least 1997 (Byman et al. 1999). Under the BWC, member nations agree to not aid the biological weapons programs of foreign nations, thereby making China's past trade actions noncompliant with the BWC. However, the BWC lacks mechanisms to enforce compliance and to investigate and respond to events of noncompliance.

Nevertheless, for almost fifty years the BWC has been the gold standard for watching for and preventing state-sponsored biological weapons. Advancements in synthetic biology have decreased the amount of time, money, and skill needed for an individual or organization to participate in the field, leading to the development of pathogens by smaller sub-state and non-state actors who are not restricted by the BWC (Evans and Selgelid 2015), as well as potentially by individuals with relatively simple scientific knowledge. For example, al-Qaeda and Aum Shinrikyo have both attempted to develop bioweapons, although with limited success (Tucker 
2007). In the 2001 "Amerithrax" bioterror attack, an individual actor sent Bacillus anthracis spores through the U.S. Postal Service, resulting in five deaths, the prophylaxis of 30,000 more individuals, and hundreds of millions of dollars in decontamination expenses (Pita and Gunaratna 2010). The destructive potential of synthetic biology is only increasing with scientific advances in the field. In the future, threats could come from disgruntled employees of state-of-the-art scientific laboratories or vengeful academics, but also simply from a sorcerer's apprentice, insufficiently prudent and educated to prevent accidents. Therefore, as the newest developments in genetic engineering grow increasingly accessible to private citizens, the question arises as to what rate-limiting steps (e.g. technical knowledge or inspiration) can prevent non-state small group or individual actors from undertaking development of increasingly advanced biological weapons.

Indeed, now the average person interested in synthetic biology can set up a lab in their home, using common household items and equipment or kits ordered over the internet, in a movement now known as "DIY Bio" ("do-it-yourself biology") or "garage biology" (Ledford 2010). Additionally, while chemicals have a set structure and formula that makes them easier to identify and govern, advancements in synthetic biology allow for the creation of novel types of biological weapons that are not explicitly prohibited by the BWC but instead exist in a sort of "gray zone." This increased accessibility of synthetic biology and difficulty in categorizing synthetic biology products creates a threat space that is not fully captured within the current structure or implementation of the BWC.

\subsection{Effective Governance and Policy for Biosecurity}

Because emerging biotechnologies are dual-use, governance must weigh the risk of misuse with the potential for beneficial use in innovation and development. Unfortunately, biosecurity attempts are mired in uncertainty around both the actual capabilities of synthetic biology, as well as the motivations of actors given the increasing number of contexts in which synthetic biology is used. Modern governments are still relying on old rules to regulate a new technology, clearly an insufficient strategy for ensuring security in the coming decades.

Building an effective biosecurity strategy to encompass twenty-first century biotechnologies requires understanding the novelties that sciences like synthetic biology create in the biosecurity threat space, as well as the structural vulnerabilities these sciences can exploit and the likely causes of inadequate biosecurity practices. Synthetic biology's novel biosecurity concerns arise from its broad scope, wider availability, complexity, and uncertainty over current and future capabilities. For example, critical developments such as gene editing via CRISPR gene editing vastly improve upon previous genetic engineering processes and may yield a revolution in human and environmental health research, but may also cause substantial and irreversible harms. One application of gene editing is the gene drive, which can rapidly propagate a certain set of genes or alleles through a population, circumventing 
Mendelian inheritance laws and increasing the chance that this set of genes is passed on. While gene drives are an exciting new technology, their ability to rapidly alter the genetic makeup of a population is cause for concern. Other potential negative consequences of gene editing may include the unconstrained diffusion of geneedited material throughout the environment, the disruption of ecologies with genetically-modified organisms (in particular engineered gene drive systems), and off-target impacts from genome editing. These techniques could also be used maliciously, with an actor purposely targeting humans and/or the environment.

The publication and dissemination of a methodology for synthesizing horsepox in a laboratory setting was a recent application of gene editing (Noyce et al. 2018). Some critics say this information could support a nefarious actor to reconstitute and develop smallpox, or to synthetize other viruses. Additionally, the widely publicized recreation of the 1918 Spanish Influenza (Tumpey et al. 2005), which killed some 50 million people worldwide at the close of the First World War, could facilitate the synthesis process for actors wishing to cause harm. Even nonpathogenic approaches have been described as dual-use research, ranging from the disruption of local ecologies via gene drives to the manipulation or destruction of inorganic materials.

These and dozens of other cases demonstrate the increasing ease with which an actor can acquire information and apply existing tools to deploy advanced genetic engineering applications with limited to no oversight. In 1975, the U.S. National Institute of Health ("NIH") established compliance measures for genome engineering that were enforced through funding restrictions; however, many synthetic biology innovators can now operate without NIH funding, approval, or even awareness, and NIH does not oversee research in other countries. Today, the financial costs, time limitations, and skill requirements needed to wield synthetic biology tools have scaled down such that some of these tools have become accessible even to elementary school students. Furthermore, the requisite baseline knowledge diminishes over time as synthetic biology processes become more streamlined. While such broad access to sophisticated genetic engineering knowledge and equipment can accelerate scientific breakthroughs, it also places the responsibility for biosecurity on a near infinite number of unsupervised actors across the globe.

In 2018, the BWC secretariat noted that increased access to technologies such as gene editing, gene drives, and gene synthesis is available to actors with limited or no oversight from established industry or governmental organizations, raising concerns about potential violations of the BWC. It is helpful to forecast and understand looming threats and potential mitigation strategies at various scales, but international treaties are not structured to oversee bottom-up efforts related to the localization and globalization of synthetic biology below the national scale. One part of the solution may be the broadening of engagement from established oversight agencies like NIH.

An additional option is the Responsible Research and Innovation ("RRI") approach, utilized by the UK and the EU, which appraises the potential effects of new research on society and the environment in order to improve the alignment of processes and expected outcomes with societal values and needs. RRI approaches include experts from a range of different fields whose role it is to assess scientific 
development with the goal of mitigating risk, making research advances accessible through fair and sustainable means, and upholding key morals and values. Programs that adopt the RRI approach are not meant to prevent research or the publication of results, but rather to minimize downstream harms that could make developers, companies, and/or governments liable for costly insurance and cleanup efforts. RRI becomes an important criteria in access to public funding but is not a regulatory requirement.

Biosecurity could also indicate to the general public that certain synthetic biology products have been filtered to guarantee beneficial uses. The US Nuclear Regulatory Commission (nrc.gov), for instance, performs this role for research and test reactors; the synthetic biology field would benefit from a similar regulatory body for biosecurity.

Where top-down governance proves insufficient, other actors such as universities, non-profits, and companies will need to engage their own gatekeeping and watchdog capabilities to protect against nefarious actors. Top-down governance may support such initiatives, which will require harmonization and communication up to the international level. These initiatives will need to be incentivized. Currently, though, biosecurity is viewed as an obligation, such that individuals, organizations, and companies must use their own funds to observe unstated and often confusing or contradictory needs for overall security. This balance of costs and benefits is insecure, and as such, institutions tend to want to minimize expenditures associated with oversight (Gillum et al. 2018). The best argument to support investing in biosecurity is that the advancement of synthetic biology ultimately requires public approval, whereas currently the public remains quite skeptical (Pauwels 2013; Oliver 2018). The public could grow more opposed to synthetic biology were the public inadvertently exposed to some harm as a consequence of insufficient or inadequate oversight. Biosecurity necessitates a strategy which incentivizes managers and corporations to stay up-to-date with the latest risks and concerns.

Some corporations are aware of the risks to their bottom line should the public be exposed to harm arising from a synthetic biology product. The majority of DNA synthesis companies, in fact, have joined the International Gene Synthesis Consortium ("IGSC"), whereby they utilize company resources to monitor customers and their requests for potential security problems. They are aware that implementing such biosecurity measures is in their best interests, even though there is no legal regime requiring them to do so. Likewise, in January 2020, the World Economic Forum and the Nuclear Threat Initiative published a report recommending that a technical consortium be set up in order to create a common DNA sequence screening mechanism. This screening mechanism would be based on work done by the IGSC.

Viewing longstanding biosecurity policy practices through the lens of risk analysis results in the conclusion that there are significant gaps in biosecurity effectiveness for synthetic biology. These inefficient and inadequate policies include (a) viewing security as a cost or undesirable expense to be minimized, (b) the siloing of scholarship and practice across disciplinary domains and among government, industry, academia, and civil society, and (c) the narrow framing of security 
problems ignores new actors and technological developments taking place in a variety of different countries and in adjacent technology fields. Each of these issues could be resolved through policy solutions that both encourage technological development and mitigate security threats while enabling public engagement in synthetic biology and investment in its products as they enter the marketplace. Policies for synthetic biology must be scalable, transferable, and adaptable in order to take into account its emerging technical and social challenges.

The increasingly globalized, distributed, and dispersed nature of synthetic biology products and research worsens challenges arising from differing practices of biosecurity governance globally. Advanced biological research is no longer overwhelmingly dominated by Europe and the US, and this may introduce different approaches to, or priorities for, biosecurity. Russia's Federal Research Programme for Genetic Technologies Development for 2019-2027, for instance, intends to "implement a comprehensive solution to the task of the accelerated development of genetic technologies, including genetic editing; to establish scientific and technological groundwork for medicine, agriculture and industry; to improve the system of preventing biological emergencies and monitoring in this area" (Ministry of Science and Higher Education of the Russian Federation 2019). Similarly, Saudi Arabia is funding research related to the development of microbial cell factories to produce fuels and chemicals, while the Singaporean government is investing considerable resources into the funding of life and environmental sciences researchat Nanyang Technological University, the National University of Singapore, and the Agency for Science, Technology and Research (A*STAR). The Chinese Academy of Sciences is establishing an Institute of Synthetic Biology, which is tasked with the dual responsibilities of fostering roadmaps for the future development of Chinese synthetic biology while also establishing safety and security norms for researchers at Chinese institutions. There are no top-down efforts beyond existing mechanisms like the BWC or the CWC (The Convention on the Prohibition of the Development, Production, Stockpiling and Use of Chemical Weapons and on their Destruction) that standardize global governance and usage of synthetic biology, and bottom-up efforts are not coordinated in their reach or messaging.

Relative newcomers to the development of synthetic biology may possess differing tolerances and constructions of risk compared to more established technology developers. The implications of the entry of such newcomers to the field, though vast, can be grouped into two general areas. One includes diverging safety and security practices at various points of an international supply chain that forms the backbone of an increasingly globalized economy. Another includes the potential for small-scale experiments or national biosecurity policies to escape the given actor's control and spill across political boundaries. While one country may find the environmental risk of a particular synthetic biology application acceptable, its spread across borders into another country may disrupt those local ecologies (i.e., crashing or hardening a particular species through genetic engineering) or expose vulnerable human populations to irreversible consequences without options for amelioration. The nature of certain synthetic biology applications (i.e. gene drives) makes it impossible for risk-averse countries to wholly quarantine themselves from exposure 
to harms resulting from another country's decisions. This is also an issue of equity given that risk-tolerant countries will reap the rewards of risks when beneficial technologies emerge, but risk-averse countries may bear their neighbors' risks without any means to capture potential rewards.

An environment of competing and incongruent risk architectures causes individual states, organizations, or industries to arrive at differing definitions of security threats or acceptable levels of loss in pursuit of a technology's intended gains. For a technology as uncertain as synthetic biology, this policy divergence may set governments, companies, and other research organizations down vastly differing policy paths, and impede consensus in assessing the minutiae of technical risk concerns or assessment protocols, or ensuring security for anyone.

\subsection{Conclusion}

Synthetic biology is a transformative technology with the possibility to change the world to the same extent as - if not more than - the digital revolution. As is the case with previous scientific breakthroughs, the potential for its dual-use and misuse represents a global problem, and necessitates that the highest levels of policy makers pay it close attention. Although targeted countermeasures can go some way toward providing protection, preventative actions are likely to be more effective given the heightened uncertainty of the field's future (Trump et al. 2020a). It is necessary that biosecurity policies and practices be updated to take into account both the unprecedented challenges associated with synthetic biology and the globalized, diffuse, and varied nature of its threat space.

Effective global biosecurity will not happen quickly, nor will it be enthusiastically adopted by all national governments and non-governmental organizations. Incentives to misuse synthetic biology with harmful consequences remain high for certain negligent actors, as are the incentives to dual-use by nefarious actors, and the coming years may see such events affecting human or environmental health. Successful biosecurity implementation must be adaptable to quickly incorporate uncertainty as well as new capabilities. Urgent steps are required to place such notions into practice before a major threat incident, which in addition to creating substantial damages could usher in policy changes that might limit or ban platforms of synthetic biology research entirely. Besides forgoing the benefits of the technology, such a ban could force development underground and further out of the reach of coordinated governance or risk assessment. By identifying the social, economic, institutional, and technological tripwires that influence a state's trajectory towards biosecurity research of concern, now is the time to take steps to apply biosecurity to maximize technological benefits while minimizing the dual-use potential of synthetic biology by improving the framing, prioritization, and governance of biosecurity risks.

Many individuals and organizations are already taking on the challenge of biosecurity. For instance, the International Genetically Engineered Machine (iGEM) 
synthetic biology competition requires that judges carry out rigorous reviews of each team's planned experiments and the materials they will require. Additionally, iGEM's Safety and Security Committee as well as a commercial partner review team plans for potential harms (McNamara et al. 2014; Millet et al. 2019).

Biosecurity is prioritized by a number of independent and governmental organizations, including the DIY biology movement, which has a code of ethics operative in North America and in Europe (DIYbio.org). This prioritization is also indicated by the intent of the member states of the BWC to develop a code of conduct (Meeting of the States Parties 2018), the African Union's development of biosecurity norms for transgenic insects and genetically-modified crops (Glover et al. 2018), and the MIT Bio Summit 2.0 (www.biosummit.org) statement of shared purpose. Additionally, there is increasing demand for an update to global biosecurity practices and norms along the lines of the Cartagena Protocol on Biosafety. Such an update should improve cooperation, transparency, and joint security in the practice of synthetic biology.

Still, the problem of how to incentivize private actors to invest in biosecurity remains. The answer will require the participation not only of bench scientists, but also of various overseers, gatekeepers, and watchdog groups involved in biotechnology research and development (for instance, the World Organisation for Animal Health's Guideline for Responsible Conduct in Veterinary Research). One example of a potential approach is to train journal editors to recognize potential information hazards within article submissions. Additionally, funders responsible for reviewing grants could require that applicants include a review of potential information and security hazards which might occur over the course of the proposed work. In these and other examples, a fusion of top-down and bottom-up approaches is necessary in order to identify security threats and to raise awareness of biosecurity issues; meanwhile, bottom-up organizations can develop on-the-ground passive surveillance programs to monitor potential dual-use security threats.

An example of this collaboration is between the US Federal Bureau of Investigation (FBI) and iGEM. The FBI has funded iGEM and collaborated with the competition's organizers in order to increase awareness of risk and to build an understanding of possible or developing threats. While no biosecurity policy or practice can completely eliminate all threats (especially not without unilaterally preventing research and innovation that could greatly benefit society), a layered approach in which government, private organizations, and individual citizens collaborate will result in a more unified effort for biosecurity which could reduce gaps in oversight that might be exploited by actors looking to develop biological weapons.

Ultimately, adapting national and global biosecurity principles and practices may yield a number of downstream benefits that will help emerging biotechnologies become safer, more responsibly available, insurable, and trusted by key stakeholders and the broader public. Actions to achieve such goals, such as promoting multistakeholder discourse for top-down and bottom-up governance, increased global coordination, and crafting and implementing mechanisms that foster effective, sustainable, and adaptive biosecurity, are likely needed to bridge the gap between earlier generation biotechnology policy and the cutting-edge scientific and technological 
capabilities of the next decade (Trump et al. 2020a, b). This book includes a number of international opinions and scholarship on how such goals might be achieved, building from insights related to governance, risk assessment, ELSEI (ethical, legal, social and environmental issues), forecasting and horizon scanning, and the state of the art in physical and life sciences. While solutions for biology will be difficult to achieve due to a number of competing incentives, political challenges, and institutional requirements, we believe that the ideals expressed herein help signal how improved biosecurity might be achieved over time.

To explore the various topics pertaining to emerging biosecurity challenges, the chapters that follow build from a NATO Science for Peace and Security Programme (SPS) Advanced Research Workshop (ARW) hosted by the École Polytechnique Fédérale de Lausanne (EPFL) in Lausanne, Switzerland in July 2019. Specifically, participants from over two dozen countries gathered to explore various physical and life sciences as well as social sciences concerns related to emerging biotechnology and associated security needs, challenges, and opportunities. This book includes written perspectives from the various working groups, including (a) top-down governance at the national and international levels, (b) bottom-up governance using grassroots and self-governing interests, (c) the uncertain and difficult challenges posed by information hazards, (d) security concerns from technical, life sciences, and computer-assisted design research, and (e) the approaches and challenges of foresight in the forecasting of future threats, opportunities, and governance needs within the broader biosecurity space (Linkov et al. 2018). Additional chapters emphasize more explicit topics within each of these core ideas, and elucidate different perspectives on how to better understand the limitations of modern biosecurity, as well as how it may be improved from a risk assessment, governance, and multidisciplinary sciences perspective.

\section{References}

Ahteensuu M (2017) Synthetic biology, genome editing, and the risk of bioterrorism. Sci Eng Ethics 23:1541-1561. https://doi.org/10.1007/s11948-016-9868-9

Byman D, Cliff R, Saunders P (1999) US policy options toward an emerging China. Pac Rev 12(3):421-451

Convention on the Prohibition of the Development, Production and Stockpiling of Bacteriological (Biological) and Toxin Weapons and on Their Destruction. Biological and Toxin Weapons Convention Website. 10 April 1972. http://www.opbw.org/

Evans NG, Selgelid MJ (2015) Biosecurity and open-source biology: the promise and peril of distributed synthetic biological technologies. Sci Eng Ethics 21:1065-1083. https://doi. org/10.1007/s11948-014-9591-3

Franconi R, Illiano E, Paolini F, Massa S, Venuti A, Demurtas OC (2018) Rapid and low-cost tools derived from plants to face emerging/re-emerging infectious diseases and bioterrorism agents. In: Defence against bioterrorism. Springer, Dordrecht, pp 123-139

Getz LJ, Dellaire G (2018) Angels and devils: dilemmas in dual-use biotechnology. Trends Biotechnol 36(12):1202-1205

Gillum D, Carrera LAO, Mendoza IA, Bates P, Bowens D, Jetson Z, Maldonado J, Mancini C, Miraldi M, Moritz R (2018) The 2017 Arizona biosecurity workshop: an open dialogue about biosecurity. Appl Biosaf 23:233-241. https://doi.org/10.1177/1535676018781854 
Glover B, Akinbo O, Savadogo M, Timpo S, Lemgo G, Sinebo W, Akile S, Obukosia S, Ouedraogo J, Ndomondo-Sigonda M (2018) Strengthening regulatory capacity for gene drives in Africa: leveraging NEPAD's experience in establishing regulatory Systems for Medicines and GM crops in Africa. BMC Proc 12(Suppl 8):11. https://doi.org/10.1186/s12919-018-0108-y

Gronvall G (2015) Biosecurity: the opportunities and threats of industrialization and personalization. Bull At Sci 71(6):39-44

Gronvall GK (2018) Safety, security, and serving the public interest in synthetic biology. J Ind Microbiol Biotechnol 45:463-466. https://doi.org/10.1007/s10295-018-2026-4

Ledford H (2010) Life hackers. Nature 467(7316):650

Linkov I, Trump BD, Anklam E, Berube D, Boisseasu P, Cummings C, Ferson S, Florin M-V, Goldstein B, Hristozov D (2018) Comparative, collaborative, and integrative risk governance for emerging technologies. Environ Syst Decis 38:170-176. https://doi.org/10.1007/ s10669-018-9686-5

Mayor A (2003) Greek fire, poison arrows \& scorpion bombs: biological and chemical warfare in the ancient world. Overlook Duckworth, Woodstock. ISBN:978-1-58567-348-3

McNamara J, Lightfoot SB-Y, Drinkwater K, Appleton E, Oye K (2014) Designing safety policies to meet evolving needs: iGEM as a testbed for proactive and adaptive risk management. ACS Publications, Washington, DC

Meeting of the States Parties (2018) Meeting of experts on review of developments in the field of science and technology related to the convention: reflections and proposals for possible outcomes submitted to the meeting of the states parties. Switzerland, Geneva

Millett P, Binz T, Evans SW, Kuiken T, Oye K, Palmer MJ et al (2019) Developing a comprehensive, adaptive, and international biosafety and biosecurity program for advanced biotechnology: the IGEM experience. Appl Biosaf 24(2):64-71

Ministry of Science and Higher Education of the Russian Federation. Approval of the Federal Research Programme for Genetic Technologies Development for 2019-2027. The Russian Government: Government Decisions Website. 22 April 2019. http://government.ru/en/ docs $/ 36457 /$

National Academies of Sciences, Engineering, and Medicine (2018) Biodefense in the age of synthetic biology. National Academies Press, Washington, DC. https://doi.org/10.17226/24890

Noyce RS, Lederman S, Evans DH (2018) Construction of an infectious horsepox virus vaccine from chemically synthesized DNA fragments. PLoS One 13(1):e0188453. https://doi. org/10.1371/journal.pone. 0188453

Oliver A (2018) Behavioral economics and the public acceptance of synthetic biology. Hastings Cent Rep 48:S50-S55

Pauwels E (2013) Public understanding of synthetic biology. Bioscience 63:79-89. https://doi. org/10.1525/bio.2013.63.2.4

Pita R, Gunaratna R (2010) Anthrax as a biological weapon: from World War I to the Amerithrax investigation. Int J Intell Counterintell 23(1):61-103

Trump BD, Cummings CL, Kuzma J, Linkov I (2020a) Synthetic biology 2020: frontiers in risk analysis and governance. Springer, Cham

Trump BD, Galaitsi SE, Appleton E, Bleijs DA, Florin MV, Gollihar JD et al (2020b) Building biosecurity for synthetic biology. Mol Syst Biol 16(7):e9723

Tucker JB (2007) War of nerves: chemical warfare from World War I to Al-Qaeda. Anchor, New York

Tumpey TM, Basler CF, Aguilar PV, Zeng H, Solorzano A, Swayne DE, Cox NJ, Katz JM, Taubenberger JK, Palese P, Garcia-Sastre A (2005) Characterization of the reconstructed 1918 Spanish influenza pandemic virus. Science 310(5745):77-80. https://doi.org/10.1126/ science. 1119392

Yeh JY, Seo HJ, Park JY, Cho YS, Cho IS, Lee JH et al (2012) Livestock agroterrorism: the deliberate introduction of a highly infectious animal pathogen. Foodborne Pathog Dis 9(10):869-877

Zilinskas RA (2012) Take Russia to 'task' on bioweapons transparency. Nat Med 18(6):850-850 
Open Access This chapter is licensed under the terms of the Creative Commons Attribution 4.0 International License (http://creativecommons.org/licenses/by/4.0/), which permits use, sharing, adaptation, distribution and reproduction in any medium or format, as long as you give appropriate credit to the original author(s) and the source, provide a link to the Creative Commons license and indicate if changes were made.

The images or other third party material in this chapter are included in the chapter's Creative Commons license, unless indicated otherwise in a credit line to the material. If material is not included in the chapter's Creative Commons license and your intended use is not permitted by statutory regulation or exceeds the permitted use, you will need to obtain permission directly from the copyright holder. 